\section{Massive PANNICULECTOMY: A NOVEL METHOD OF TREATMENT OF POSTLAPAROTOMY WOUND DEHISCENCE IN MORBID OBESITY}

It was with interest that we read the article "High-concentration oxygen and surgical site infections in abdominal surgery: a meta-analysis." Massive liposuction and body contouring procedures are no longer primary therapeutic options for the treatment of morbid obesity owing to their complications and suboptimal results. The obvious paradigm shift has been noted to favour bariatric procedures since the advent of laparoscopic approaches over last 2 decades. Panniculectomy is a cosmetic procedure that involves the removal of excess skin and fat from the affected organ.

We performed massive panniculectomy for the treatment of postlaparotomy wound dehiscence in a morbidly obese patient. A 60 -year-old woman with body mass index (BMI) of 41.7 underwent a total abdominal hysterectomy for dysfunctional uterine bleeding. A surgical site infection and dehiscence involving skin and subcutaneous layers across the complete length of incision developed in the immediate postoperative period. The initial treatment at the primary centre involved drainage, debridement and regular dressings.

The patient was referred to our centre for further management in postoperative week 4. Upon clinical examination, we observed that the abdomen skin fold was thick and large, and it was difficult to examine the dehiscence suture line in the recumbent position (Fig. 1A). The bacteriological study of culture and sensitivity from the abdominal wound was unremarkable; blood work was essentially normal. After counselling the patient and her relatives, a decision was made to repair the wound with massive panniculectomy to remove excessive skin and subcutaneous tissue from the abdomen. The excessive abdominal skin complex incorporating

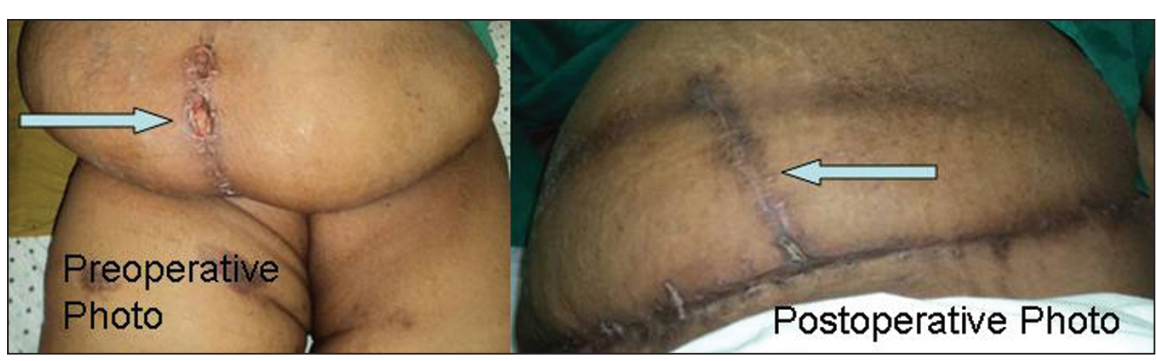

Fig. 1. (A) Clinical picture showing laparotomy wound dehiscence. (B) Postoperative picture of the suture line shows good healing after massive panniculectomy surgery.

the suture line and the umbilicus was removed (weighing $8.5 \mathrm{~kg}$ ) and the procedure was completed. The duration of the surgery was 180 minutes; blood loss was minimal $(100 \mathrm{~mL})$. The postoperative period was uneventful and the wound healed well, with no excessive abdominal fold. The patient was able to resume her normal activities 14 days after the procedure (Fig. 1B).

Management of morbid obesity involves a trial of strict diet and exercise, followed, if unsuccessful, by one of the various bariatric surgical procedures. Plastic surgical intervention usually comprises massive liposuction and body contouring with or without lumpectomies after achieving weight loss.

Panniculectomy is a procedure that involves the removal of excess skin and fat from any part of the body. This procedure is different from abdominoplasty, which involves tightening of the muscles. Panniculectomy is done routinely after massive weight loss (e.g., after bariatric surgeries), where excessive skin and subcutaneous fat are removed to enhance appearance and improve confidence. When more than 10 lbs of tissue is removed, the procedure is known as massive panniculectomy. ${ }^{2,3}$

Abdominal wall hernia repair is treated with mesh repairs, component separation technique and pedicled fascia lata flap. In our case, excess bulk of the abdominal wall mass was dragging in multiple directions, thereby stretching the operative wound and preventing it from closing. During the procedure, the previous vertical wound was excised en masse with the excess abdominal apron and umbilicus using a supra pubic Pfannensteil incision; the postexcision wound was closed transversely corresponding to the relaxed skin tension lines. Therefore, a reduced abdominal wall mass and a new suture line along the relaxed skin tension lines enabled us to have good wound healing and increased patient satisfaction.

We conclude that panniculectomy, which is primarily a cosmetic method of excess skin and fatty tissue removal, can be used to treat postsurgical wound dehiscence in morbidly obese patients.

\author{
Abhijeet Ashok Salunke, MBBS, DNB \\ (Ortho) \\ Assistant Professor \\ Department of Orthopedics \\ Sri Krishna Hospital \\ Pramukswami Medical College \\ Gujarat, India \\ Clinical Fellow \\ Orthopedic Oncosurgery \\ National University Hospital \\ Singapore
}

K. S. Rajkumar, MS, FRCS

Department of General Surgery and Laparoscopic Services

Kovai Medical Center and Hospital

Coimbatore, Tamilnadu, India

G.I. Nambi, MS, MCh (Plastic Surgery)

Department of Plastic and Reconstructive

Microvascular Services

Kovai Medical Center \& Hospital

Coimbatore, Tamilnadu, India

Clinical Fellow

Hand and Reconstructive Microsurgery

National University Hospital

Singapore

Vikram Anil Chaudhari, MBBS, DNB (GI Surgery)

Department of Surgical Gastroenterology

Seth GS Medical College and KEM Hospital

Mumbai, Maharashtra, India

Competing interests: None declared.

DOI: $10.1503 /$ cjs.029213 


\section{References}

1. Patel SV, Coughlin SC, Malthaner RA. High-concentration oxygen and surgical site infections in abdominal surgery: a meta-analysis. Can 7 Surg 2013;56:E82-90.

2. Manahan MA, Shermak MA. Massive panniculectomy after massive weight loss. Plast Reconstr Surg 2006;117:2191-7.

3. Meyerowitz BR, Gruber RP, Laub DR. Massive abdominal panniculectomy. FAMA 1973;225:408-9.

\section{ANCILLARY TESTS TO IMPROVE THE ACCURACY OF LAPAROSCOPY IN THE DIAGNOSIS OF TUBERCULOUS PERITONITIS}

It has been suggested in your journal that laparoscopy and biopsy should be deployed to diagnose tuberculous peritonitis. ${ }^{1}$ In 1993, the World Health Organization took an unprecedented step and declared tuberculosis (TB) a global emergency. ${ }^{2}$ The prevalence of extrapulmonary $\mathrm{TB}$ is rising, possibly related to coinfection with HIV. In patients with extrapulmonary $\mathrm{TB}$, the abdomen is involved in $11 \%$ of patients. ${ }^{2}$ Tuberculous peritonitis is believed to exhibit a female predominance; other causes of abdominal pain may delay diagnosis.

Over a 2-year period, we carried out a prospective study of 92 patients admitted to the Government Medical College at Srinagar with the clinical suspicion of abdominal TB. Of these patients, 50 were subsequently proven by biopsy to have peritoneal $\mathrm{TB}$.

All the patients underwent the following diagnostic tests after a thorough clinical examination: erythrocyte sedimentation rate (ESR); Mantoux test; ascitic fluid analysis for adenosine deaminase (ADA) levels, Ziehl-Neelsen staining and culture of mycobacterium tubercle bacilli (MTB); and contrast enhanced computed tomography (CT) of the abdomen. We then undertook diagnostic laparoscopy. Features of a positive laparoscopy included thickened peritoneum, adhesions and peritoneal tubercles, which were biopsied (Fig. 1). Our patients were mainly young adults from rural areas, with a slight female predominance. Patients presented with fever $(50 \%)$, weigh loss (40\%), aneroxia (74\%), malaise/pain (80\%), diarrhea (10\%), vomiting (40\%) and constipation (25\%). Physical signs were palor (74\%), tender abdomen (70\%), abdominal distension (50\%), abdominal mass (20\%), ascitis (15\%) and lymphadenopathy (8\%). The Mantoux test was positive in 23 patients (46\%). Anaemia was present in $84 \%$ of patients and the ESR was high in $90 \%$.

Diagnostic laparoscopy was positive in 46 of 50 patients with biopsyproven peritoneal $\mathrm{TB}$, with a sensitivity of $92 \%$. Contrast enhanced CT of the abdomen detected 30 of 50 cases of abdominal TB with a sensitivity of $60 \%$ and specificity of $71.42 \%$. ZiehlNeelsen staining for MTB of ascitic fluid was positive in only 2 patients (4\%) and culture for MTB was positive in 8 cases $(16 \%)$, whereas ascitic fluid polymerase chain reation (PCR) for TB increased the sensitivity to 83.33\%. Ascitic fluid analysis for ADA (> $33 \mathrm{U} / \mathrm{L}$ ) showed a sensitivity and specificity of $100 \%$ and $96 \%$, respectively. Diagnostic laparoscopy was falsely positive in 10 of 42 patients who did not have TB peritonitis.

Difficulties in the diagnosis of TB peritonitis owe to its nonspecific associated clinical features, unhelpful laboratory tests and a high level of falsenegative tests (i.e., Mantoux test, Ziehl-Neelsen staining and culture of ascitic fluid and false-negative imaging). High levels of ascitic ADA is associated with TB peritonitis. ${ }^{3}$ Ascitic fluid was found clinically in only $15 \%$ of our patients, but may be available at the time of operation. Histopathology of peritoneal lesions is appropriate for diagnosing $\mathrm{TB}$ and to rule out other diseases, such as cancer. In the past, this required laparotomy ${ }^{4}$ even though the potential for laparoscopy was known before the advent of video laparoscopy. ${ }^{5}$

We agree with the previous report ${ }^{1}$ on the value of laparoscopy, but we found a false-positive rate of $18 \%$. We

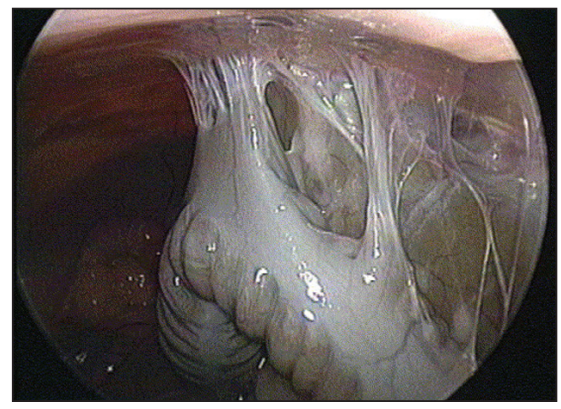

Fig. 1. Adhesions between the colon and the parietal peritoneum in a patient with tuberculous peritonitis.

suggest that the laparoscopic diagnosis of TB peritonitis be confirmed by histopathology of peritoneal lesions or ascitic fluid analysis for ADA greater than $33 \mathrm{U} / \mathrm{L}$, depending on the local availability of these tests.

\section{Mumtazdin Wani, MS \\ Professor \\ Department of Surgery \\ Government Medical College \\ Srinagar, India}

Shabir Ahmad Mir, MBBS, PG Scholar, Surgery

Department of Surgery

Government Medical College

Srinagar, India

Jahangeer Ahmad Bhat, MD

Department of Radiodiagnosis Government Medical College

Srinagar, India

Hakim Adil Moheen, MBA

Kashmir University

Srinagar, India

DOI: $10.1503 /$ cjs.032713

\section{References}

1. Akgun Y. Intestinal and peritoneal tuberculosis: changing trends over 10 years and a review of 80 patients. Can $\mathcal{F}$ Surg 2005;48: 131-6.

2. World Health Organization. Fact Sheet No 104: Tuberculosis [report]. 2002 Aug. Available: www.who.int/mediacentre /factsheets/who104/en/print.html (accessed 2013 Dec. 13).

3. Shen YC, Wang T, Chen L, et al. Diagnostic accuracy of adenosine deaminase for tuberculous peritonitis: a meta-analysis. Arch Med Sci 2013;9:601-7.

4. Wells AD, Northover JM, Howard ER. Abdominal tuberculosis: still a problem today. F R Soc Med 1986;79:149-53.

5. Wolfe JH, Behn AR, Jackson BT. Tuberculous peritonitis and role of diagnostic laparoscopy. Lancet 1979;1:852-3. 